\title{
Continuing medical education (CME) or continuing professional development (CPD): a need, a challenge, but also a must
}

\author{
Andrés Cervantes*
}

$\mathrm{T}$ he rate at which medical knowledge is increassing is almost overwhelming. Basic science is creating new and complex paradigms, used to better understand biological facts and functions, which are challenging our interpretation of many clinical events. All these new findings will eventually have an impact on clinical practice. If we look back to the last twenty years, there are many medical disciplines that have moved completely from relative technical simplicity to a changing scenario where many diverse and complex tools are now used to provide better care to our patients. Think about imaging, pathology or even the basis of therapy and consider how different they were only two decades ago. But perhaps the most striking issue is that this moving picture still has a long way to go.

When we apply this framework to cancer medicine, the facts are even more striking. For example, our current understanding of breast or lung cancer requires the implementation of several diagnostic and therapeutic tools that guide clinicians to a more efficient decision-making process. Could you imagine yourself nowadays deciding any therapy for a new breast cancer patient without knowing the hormonal receptor or HER2 status?

Apart from technical developments, most of the knowledge and capabilities needed by doctors to adapt to current standards are acquired during their practice as physicians and not while obtaining their MD degree. Having a well designed continuing medical education (CME) program is not only a need and a challenge, but a must for physicians. Oncologists are confronted every day with an overwhelming amount of information, coming not only from scientific and peer-reviewed journals, but also from the lay press. It

\footnotetext{
A. Cervantes ( $\triangle)$

Department of Hematology and Medical Oncology

Hospital Clínico Universitario

University of Valencia

Valencia, Spain

e-mail: andres.cervantes@uv.es

*Andrés Cervantes is serving at the moment as a member of the Steering Committee of the Educational Committee of ESMO and as Chair for the E-learning and CME Working Group.
}

is not uncommon to get the final results of relevant clinical trials published in high impact factor scientific journals at the same time as in well known and widely distributed newspapers.

The importance of CME is clear. We all may agree that any oncologist can carry out his or her work properly without keeping up to date with relevant advances that are commonly generated by clinical, translational and basic research. On the other hand, it is not difficult to understand why Society should protect their members by trying to guarantee that all practising doctors have the required knowledge and capabilities to offer optimal clinical care according to well established standards.

The absence of a common framework for CME in Europe leads to different strategies in every individual country with markedly different approaches and aims. In Spain CME is a voluntary process and all obtained CME credits are used as an incentive for professional careers of physicians employed by the 17 regional health services. However, it does not have any influence on recertification, revalidation or relicensing. This is not the case in many other European countries, such as Switzerland, Germany, Ireland or the United Kingdom, where CME is mandatory for recertification. For example, in Germany, every doctor must collect $250 \mathrm{CME}$ credits over a five-year period and have proof of them to obtain the approval of the regional chambers of physicians, who are responsible for issuing the final CME certificate. In the same system, the Federal Medical Association works on harmonisation and mutual recognition of CME credits. However, despite such a diverse landscape, some efforts are being made to create recognition of $\mathrm{CME}$ accreditation across Europe and to harmonise credits and practices.

In the process of CME we should consider several partners: the provider, the learner and the national body that guarantees the independence and quality of the process. In Spain, medical societies or cooperative groups provide about $50 \%$ of CME opportunities, while employers (20\%), pharma companies $(15 \%)$ and private institutions $(10 \%)$ provide the rest. Of note, universities only provide less than $5 \%$ of the whole amount of CME opportunities. However, pharma companies are the main financial backers, covering more than two thirds of the total cost of those CME events while employers (20\%) or medical societies (10\%) cover the rest. 
Concerning the learners, it is important that any CME event is oriented not only towards teaching new findings in the sense of being informative. The expansion of academic knowledge and skills is closer to the traditional conception of CME. At the very end of any educational activity of this type, the learner may have more knowledge, more experience and better skills, but this does not indicate how doctors will apply what they have learned in their clinical practice. CME certificates are not sufficient to prove doctors' competence. The only thing they prove is attendance. The main point is perhaps how to orient these CME activities in order to improve doctors' performances in daily clinical practice. More important than CME may be continuing professional development (CPD), which deals with updating, developing and enhancing how doctors apply the knowledge, attitudes and skills required in their working lives. CPD focuses on practice improvement and therefore it develops all competence levels, those of self-reflection, performance analysis, quality improvement and assurance, communication and leadership, internal and external feedback, and personal and social competency, adding to the classical concept of CME.

Last, but no least, we should consider the importance of a national body that guarantees the independence and quality of the whole CME process. The Spanish accreditation Council for CME was created in 2003 and as a national body for accreditation is responsible for registering and reviewing all the application forms presented by providers. Three external peer review experts will check the quality assessment of the proposal and after this step the Council will assign the final number of credits to the CME event.

Twenty years ago the European Society for Medical Oncology (ESMO) initiated an official examination to provide a title to be recognised all over Europe. Several countries now require that, after finishing the training period in medical oncology, all residents should pass the ESMO examination in order to get the Oncology Certification, but this requirement is not universal. Moreover, with the ESMO MORA program (Medical Oncologist's Recertification Approval) all oncologists who pass the exam can recertificate themselves every five years following at least 250 credits or by retaking the exam again. More recently the ESMO MORA working group was renamed as the e-learning and CME working group. The group also redefined its strategy and goals, focusing on e-learning activities and fostering new challenging CME opportunities.

The future of CME, as well as that of CPD, faces a great challenge. It should orient and motivate doctors in a process of adaptation of new knowledge and capabilities, which might improve the service our patients receive. Education aims to modify our behaviour, making us ready to serve in a more efficient and professional way. 